\title{
Cardio-respiratory responses of the 6-minute walk test in patients with refractory heart failure during the preoperative period for heart transplant surgery
}

\section{Risposta cardio-respiratoria al Test del cammino dei 6 minuti in pazienti con scompenso cardiaco refrattario nel periodo preoperatorio del trapianto cardiaco}

\author{
Gerson Cipriano Jr11, Graziella França Bernardelli2, Ross Arena ${ }^{3}$, \\ Luis Vicente Franco Oliveira4, Francisco Valdez, João Nelson Rodrigues Branco2
}

\begin{abstract}
Cardio-respiratory responses of the 6-minute walk test in patients with refractory heart failure during the preoperative period for heart transplant surgery. G. Cipriano Jr, G.F. Bernardelli, R. Arena, L.V.F. Oliveira, F. Valdez, J.N.R. Branco.

Background: The six-minute walk test (6MWT) has been used to assess functional capacity, clinical status and prognosis. There are a very few descriptions in the literature on the safety and metabolic impact of the test, especially in patients with severe heart failure, awaiting cardiac transplantation. Objective: The aim of the present study was to assess the cardiovascular responses and correlate the performance on the 6MWT with clinical status. Method: From 15 initial candidates, twelve patients (10 males) aged $52 \pm 8$ years were submitted to a comprehensive clinical evaluation. The patients performed the 6MWT with electrocardiographic and perceived exertion monitoring in addition to determination of blood lactate concentration. Patients were followed up for $\mathbf{1 2}$
\end{abstract}

months. Results: The patients walked $399.4 \pm 122.5$ meters, reaching a perceived exertion (PE) of $14.3 \pm 1.5$ and an increase of $34 \%$ in resting heart rate. Two patients exhibited a greater severity of arrhythmia prior to the 6MWT, which did not increase during exertion. Four patients exhibited a significant increase in blood lactate levels $(>5 \mathrm{mmol} / \mathrm{dL})$ and three interrupted the test prematurely. The distance walked (D) revealed a correlation with the ejection fraction $(\%)$ and functional classification (NYHA). After 12 months of follow up, three patients died and seven were re-hospitalized due to heart failure decompensation. Conclusion: Clinical and electrocardiographic behavior suggests that the 6MWT is safe, but may be considered of high intensity for some patients with severe heart failure. Variables related to the performance on the 6MWT may be associated to worsening clinical status in this population.

Keywords: exercise, ergometry, heart failure.

Monaldi Arch Chest Dis 2010; 74: 64-69.

\footnotetext{
1 Physiotherapy Division, Faculty of Ceilândia, University of Brasília, UnB, Brasília, Brazil.

2 Cardiovascular Surgery Division, Department of Surgery, Federal University of Sao Paulo, Sao Paulo, Brazil.

3 Department of Physical Therapy, Box 980224, Virginia Commonwealth University/MCV Campus, Richmond, Virginia, USA.

4 Rehabilitation Sciences Master's Program, Universidade Nove de Julho - UNINOVE, São Paulo, SP, Brazil.
}

Corresponding author: Gerson Cipriano Jr, Ph.D., PT.; Faculdade Ceilândia; University of Brasília - UnB; QNN 14 Área Especial Ceilândia Sul; CEP 72220-140 - Brasília - DF - Brasil; (55) (61) 3376-6042; (55) (61) 7813-2755; E-mail address: ciprianeft@uol.com.br

\section{Introduction}

Refractory heart failure (HF) is defined as a condition of persistence or deterioration of clinical condition despite intensive therapy. The HF, brought about several forms of heart disease, typically leads to a progressive deterioration of clinical status and ultimately death [1]. The failure of compensatory mechanisms to maintain cardiac output favors protein-energy malnutrition, clinically manifested by a severe reduction in functional capacity $[2,3]$. Heart transplantation is a viable therapeutic option for this group, increasing life expectancy, cardiac function and functional capacity (FC). However, after being listed as an appropriate candidate for this surgical procedure, therapeutic management in the pre-transplant phase may be delicate and taxing [4].

Cardiopulmonary exercise testing with ventilatory expired gas analysis is the preferred method for evalu- ation of functional capacity in patients with HF. However, serial assessments are logistically difficult to execute, which can hinder more detailed control of patients with this clinical condition [5]. In such cases, the six-minute walk test (6MWT) may be indicated as a simplified, reproducible, low-cost alternative for assessment of tolerance to physical exertion, providing insights into clinical status, response to interventions and quality of life, especially in patients with a more pronounced reduction in functional capacity [6-10].

Specific scientific knowledge regarding the safety and the physiologic response to the 6MWT in patients with refractory HF is limited at this time. Given the potential for a profound reduction in functional capacity and for exercise induced arrhythmias and secondary complications, particularly present in conditions of reduced myocardial oxygen supply, often encountered in this population, utilization of the 6MWT may prove to be a 
useful clinical assessment. Therefore, the aim of the present study was to assess cardiovascular response to the 6MWT and the possible correlations to clinical status in patients with refractory HF listed for transplantation.

\section{Methods}

\section{Participants in the study}

Twelve patients (aged $52 \pm 8$ years, 10 male and 2 female) receiving their care at the heart transplant clinic at the Federal University of Sao Paulo hospital were included in this study. The patients had a clinical diagnosis of refractory HF, and were considered appropriate candidates for heart transplantation. The principal $\mathrm{HF}$ etiologies were related to ischemic $(58.3 \%)$ and idiopathic (25\%) cardiomyopathy. Eight patients $(67.7 \%)$ were in functional classes III and IV (NYHA); ten (83.3\%) had associated co-morbidities; and nine $(75 \%)$ had undergone previous cardiovascular procedures (5 myocardial revascularization surgeries and 4 transluminal angioplasties) (Table 1).

Table 1. - Initial characteristics of patients in preoperative heart transplantation period submitted to the six-minute walk test $(n=12)$

\begin{tabular}{|c|c|c|c|c|}
\hline Characteristics & Mean & \pm & SD & $\mathrm{CV}(\%)$ \\
\hline Age (years) & 52 & \pm & 8.20 & 15.56 \\
\hline Weight $(\mathrm{Kg})$ & 67.83 & \pm & 12.89 & 19.01 \\
\hline Height (m) & 1.64 & \pm & 0.10 & 6.08 \\
\hline BMI (Kg/m2) & 23.50 & \pm & 4.15 & 17.64 \\
\hline $\mathrm{W} / \mathrm{H} \mathrm{R}$ & 0.97 & \pm & 0.06 & 6.51 \\
\hline Quality of Life (Minnesota QOL) & 31.00 & \pm & 3.33 & 37.21 \\
\hline Ejection fraction $(\%)$ & 26.33 & \pm & 4.51 & 17.16 \\
\hline Time since diagnosis of CHF (months) & 98.75 & \pm & 822.92 & 31.55 \\
\hline \multicolumn{5}{|l|}{ Respiratory Muscle Strength } \\
\hline $\mathrm{P}_{\mathrm{i}-\max }\left(\mathrm{cmH}_{2} \mathrm{O}\right)$ & -89 & \pm & 25.79 & 29.09 \\
\hline $\mathrm{P}_{\mathrm{i}-\max }(\%$ of predicted $)$ & 80 & $\overline{ \pm}$ & 27.23 & 34.03 \\
\hline $\mathrm{P}_{\mathrm{e}-\max }(\mathrm{cmH} 2 \mathrm{O})$ & 83 & \pm & 26.08 & 31.43 \\
\hline \multirow[t]{2}{*}{$\mathrm{P}_{\mathrm{e}-\mathrm{max}}(\%$ of predicted $)$} & 109 & \pm & 31.03 & 28.53 \\
\hline & No & & $(\%)$ & \\
\hline \multicolumn{5}{|l|}{ Gender } \\
\hline Male & 10 & & $(83.3)$ & \\
\hline \multicolumn{5}{|l|}{ Functional Class (NYHA) } \\
\hline II & 4 & & $(33.3)$ & \\
\hline III & 5 & & (41.7) & \\
\hline IV & 3 & & $(25.0)$ & \\
\hline \multicolumn{5}{|l|}{ Etiology of CHF } \\
\hline Ischemic myocardiopathy & 7 & & $(58.3)$ & \\
\hline Idiopathic myocardiopathy & 3 & & $(25.0)$ & \\
\hline Congenital heart disease & 2 & & (16.7) & \\
\hline \multicolumn{5}{|l|}{ Co-morbidities } \\
\hline Kidney failure & 1 & & (8.3) & \\
\hline Diabetes Mellitus & 1 & & $(8.3)$ & \\
\hline Myocardial infarction & 8 & & (66.7) & \\
\hline 1 event a & 2 & & $(25.0)$ & \\
\hline 2 events a & 4 & & $(50.0)$ & \\
\hline 3 events a & 1 & & $(12.5)$ & \\
\hline 4 events a & 1 & & (12.5) & \\
\hline \multicolumn{5}{|l|}{ Risk factors } \\
\hline Smoking & 7 & & $(58.3)$ & \\
\hline Arterial Hypertension & 7 & & $(58.3)$ & \\
\hline \multicolumn{5}{|l|}{ Previous heart procedures } \\
\hline Myocardium revascularization & 5 & & (41.7) & \\
\hline Use of 2 grafts $b$ & 3 & & $(60.0)$ & \\
\hline Use of 3 grafts b & 2 & & $(40.0)$ & \\
\hline Angioplasty & 4 & & $(33.3)$ & \\
\hline \multicolumn{5}{|l|}{ Medications in use } \\
\hline ACE inhibitor & 10 & & $(83.3)$ & \\
\hline Digitalis & 6 & & $(50.0)$ & \\
\hline Diuretic & 12 & & $(100.0)$ & \\
\hline$\beta$-blocker & 10 & & $(83.3)$ & \\
\hline Vasodilator & 2 & & $(16.7)$ & \\
\hline $\mathrm{Ca}^{2+}$ channel blocker & 0 & & 0.0 & \\
\hline Anti-arrhythmia agent & 1 & & (8.3) & \\
\hline Anticoagulant & 0 & & 0.0 & \\
\hline
\end{tabular}

Abbreviations: BMI, body mass index; W/H R, waist/hip ratio; NYHA, New York Heart Association; CHF, congestive heart failure; ACE, angiotensin-converting enzyme; $\mathrm{P}_{\mathrm{i}-\mathrm{max}}$, maximum inspiratory pressure; $\mathrm{P}_{\mathrm{e}-\mathrm{max}}$, maximum expiratory pressure.

Continuous parametric data represented as mean \pm standard deviation; categorical or continuous data represented as NO, number of patients $(\% \text { of total })^{a}$, frequency in 8 patients with acute myocardial infarction, b, frequency in 5 patients with revascularization of the myocardium. 
This study was approved by the local research ethics committee and informed consent was obtained from all patients prior to study.

\section{Design and protocol}

This was a prospective cohort study initially carried out on fifteen patients. Three were excluded due to cardiovascular decompensation before performed the 6MWT and twelve were submitted to the protocol, which was comprised of an initial clinical evaluation with information on clinical status, followed by the six-minute walk test (6MWT). The patients were then followed up for a period of 12 months following the 6MWT.

\section{Initial clinical evaluation}

Clinical and echocardiographic information [11, 12], such as etiology, presence of co-morbidities, previous cardiovascular procedures, clinical treatment, cardiovascular function - ejection fraction (EF) $(\%)$ and functional class (FC) (NYHA) [13] was collected for all patients. The patients were also assessed as to the impact of HF on quality of life and respiratory strength, using the Minessota Living with Heart Failure Questionare (MLHF) [14] and digital manuvacuometer.

\section{Six-minute walk test}

The patients were initially submitted to an electrocardiogram at rest to determine the existence of arrhythmias. The 6MWT was carried out in compliance with the American Association of Cardiovascular and Pulmonary Rehabilitation guidelines [15]. Specifically, the test was performed on a circular track, 30 meters in length, with no accompaniment other than standardized vocal orientations at oneminute intervals. The patients were monitored with regard to heart rate (HR, bpm), heart rate recovery at two minutes following test termination (HRR, bpm), blood pressure (BP, mmHg), peripheral oxygen saturation $\left(\mathrm{SpO}_{2}, \%\right)$ and perceived exertion (PE) using the 6-20 Borg scale [16]. Continuous variables were also monitored through electrocardiogram telemetry (QUARK T12, model CO9055-32-99, Cosmed®, Italy), enabling the instantaneous monitoring of arrhythmic events that. Peripheral lactate levels and blood glucose levels were measured prior to and following the 6MWT, was measured for each fasting subject by capillary finger-stick using the AC monitor. The basal peripheral measurement was taken after a minimum of one hour without any exercise and/or exertion and the final measurement was taken immediately upon finishing the test.

\section{2-month follow-up after the 6MWT}

The patients were followed for 12 months after performing the 6MWT in order to gather information on their clinical evolution, such as re-hospitalization secondary to $\mathrm{HF}$ decompensation and/or death.

\section{Statistical methods}

Continuous parametric data are presented as mean and standard deviation and compared over time using ANOVA for repeated measurements, with the Newman-Keuls post hoc test and the Stu- dent's t-test. Non-parametric data were represented as median and percentile and compared over time using the Friedman test, with the Muller-Dunn post hoc test and the Wilcoxon rank sum test. Categorical data are presented as absolute frequency (n) and relative frequency $(\%)$ and compared over time using McNemar's test and chi-square test (independent groups). Pearson's linear correlation coefficient was used to determine associations between the test variables and clinical status, considering the following agreement values: $>0.75$ excellent; between 0.40 and 0.75 moderate; and $<0.40$ poor. An alpha risk less than or equal to $5 \%$ and a beta risk less than or equal to $20 \%$ were considered throughout the study. All statistical tests with a p-value $<0.05$ were considered significant.

\section{Results}

\section{Occurrence of arrhythmias}

The occurrence of arrhythmias was assessed in three manners during the 6MWT. Descriptive analysis (represented by the type of arrhythmia) revealed no exacerbation during the $6 \mathrm{MWT}(\mathrm{p}=0.228)$. Likewise, qualitative analysis regarding a change in risk status during the test revealed no exacerbation, using classifications of origin $(\mathrm{p}=0.546)$ or type (0.735) (Table 2).

\section{Systemic adjustments during the 6MWT}

Unlike the $\mathrm{SpO}_{2}$ and $\mathrm{BP}$, which demonstrated no significant alterations during exertion, there was a significant increase in HR (Figure 1), proving to be the main element responsible for the positive adjustment of the double heart product (Table 3).

The average distance walked was $399.4 \pm 122.5$ meters, with an increase in perceived exertion (14 \pm 1.5 ) and evident variation in lactate level. Four individuals reached lactate levels above $4 \mathrm{mmol} / \mathrm{L}$, thereby suggesting greater use of the anaerobic metabolism during the test. Another three individuals interrupted the test prematurely - the first at $285 \mathrm{sec}-$ onds due to dyspnea, dizziness, palpitation and pain in the lower limbs; the second at 242 seconds due to pain; and the third at 180 seconds due to precordial pain. The latter two exhibited no electrocardiogram alterations characteristic of ischemia (Table 3). After interruption, the patients were allowed to continue the test until the pre-established 360 seconds had elapsed, which was when the total distance walked was recorded.

\section{Clinical status and cardiovascular follow-up}

Three patients died during the 12-month followup period - two while awaiting transplant surgery after re-hospitalization due to cardiovascular decompensation and one due to delayed rejection following cardiovascular transplant surgery. All patients were maintained under periodic outpatient follow up, but only two underwent transplant surgery during the study period, as the average time on the waiting list was $21.5 \pm 4.95$ months. During the period, six patients $(50 \%)$ manifested episodes of deterioraton in clinical status and required hospitalization five $(41.67 \%)$ for $\mathrm{HF}$ decompensation and one $(8.33 \%)$ for kidney failure. Despite of the severe 
Table 2. - Comparison of frequency, characteristic and severity of arrhythmia during the 6MWT monitored instantaneously by telemetry at rest, during and after the 6MWT in patients in the preoperative period for heart transplant surgery $(n=12)$

\begin{tabular}{|c|c|c|c|c|c|c|c|}
\hline \multirow{2}{*}{$\begin{array}{l}\text { Electrocardiogram behavior } \\
\text { Type of arrhythmia (descriptive) }\end{array}$} & \multicolumn{2}{|c|}{ Rest } & \multicolumn{2}{|c|}{ During 6MWT } & \multicolumn{2}{|c|}{ After 6MWT } & \multirow[t]{2}{*}{$p$ value } \\
\hline & No & $(\%)$ & No & $(\%)$ & No & $(\%)$ & \\
\hline Isolated extra-systole & 5 & $(41.7)$ & 6 & $(50.0)$ & 3 & $(25.0)$ & \\
\hline Unifocal ventricular extra-systole & 2 & $(16.7)$ & 2 & $(16.7)$ & 1 & $(8.3)$ & \\
\hline Divisional block & 2 & $(16.7)$ & 1 & $(8.3)$ & 1 & $(8.3)$ & \\
\hline Atrium fibrillation & 3 & $(25.0)$ & 3 & $(25.0)$ & 3 & $(25.0)$ & \\
\hline Complete block of right branch & 1 & $(8.3)$ & 1 & $(8.3)$ & 1 & $(8.3)$ & \\
\hline Sinus pause & 0 & 0.0 & 1 & $(8.3)$ & 0 & 0.0 & \\
\hline Multifocal ventricular extra-systole & 1 & $(8.3)$ & 2 & (16.7) & 2 & $(16.7)$ & \\
\hline Non-sustained ventricular tachycardia & 1 & $(8.3)$ & 0 & 0.0 & 0 & 0.0 & \\
\hline Total of patients with arrhythmia a & 9 & $(75.0)$ & 9 & $(75.0)$ & 8 & $(66.7)$ & 0.228 \\
\hline Arrhythmia severity a (based on origin) & & & & & & & 0.546 \\
\hline 0, Absent & 3 & $(25.0)$ & 3 & $(25.0)$ & 4 & (33.3) & \\
\hline 1, Supraventricular & 2 & (16.7) & 2 & $(16.7)$ & 1 & $(8.3)$ & \\
\hline 2 , Ventricular & 7 & $(58.3)$ & 7 & $(58.3)$ & 6 & $(50.0)$ & \\
\hline \multicolumn{8}{|l|}{ Arrhythmia severity (Lown classification) } \\
\hline Grade 0, no extra-systoles & 6 & $(50.0)$ & 6 & $(50.0)$ & 8 & $(66.7)$ & \\
\hline Grade $1,<30$ extra-systoles per hour & 4 & $(33.3)$ & 5 & $(41.7)$ & 2 & (16.7) & \\
\hline Grade $2,>30$ extra-systoles per hour & 0 & 0.0 & 0 & 0.0 & 0 & 0.0 & \\
\hline Grade 3, polymorphic extra-systoles & 1 & $(8.3)$ & 1 & $(8.3)$ & 2 & (16.7) & \\
\hline Grade 4A, coupled extra-systoles & 0 & 0.0 & 0 & 0.0 & 0 & 0.0 & \\
\hline Grade 4B, ventricular tachycardia $(>3)$ & 1 & $(8.3)$ & 0 & 0.0 & 0 & 0.0 & \\
\hline Grade $5, \mathrm{R}$ phenomenon on $\mathrm{T}$. & 0 & 0.0 & 0 & 0.0 & 0 & 0.0 & \\
\hline Total of arrhythmias & 0.5 & $(0-1.0)$ & 0.5 & $(0-1.0)$ & 0.0 & $(0-1.0)$ & 0.7351 \\
\hline
\end{tabular}

Abbreviation: 6MWT, six-minute walk test.

Categorical or continuous data represented as $\mathrm{NO}^{\mathrm{O}}$, number of patients (\% of total); non-parametric continuous data represented as median (percentile 25\%-75\%). a Friedman; b McNemar.

Table 3. - Characteristics and relative variations between end of exercise and rest

\begin{tabular}{lcccc}
\hline Characteristics after 6MWT & Mean & \pm & SD & CV (\%) \\
\hline Maximum distance (m) & 399.4 & \pm & 122.50 & 30.67 \\
Maximum perceived exertion (PE) & 14.3 & \pm & 1.55 & 10.86 \\
Exertion/Max. Dist. Ratio (PE/m) & 0.0 & \pm & 0.01 & 87.50 \\
Peak HR after 6MWT (bpm) & 111.4 & \pm & 25.13 & 22.56 \\
\% of HRmax (\%) & 66.3 & \pm & 12.83 & 19.34 \\
HRmax/Max. Dist. Ratio (bpm/m) & 0.1 & \pm & 0.07 & 66.22 \\
Maximum lactate after 6MWT & 3.2 & \pm & 1.04 & 32.75 \\
$\Delta$ Lactate variation, pre and post 6MWT (mmol/L) & 1.3 & \pm & 1.15 & 91.28 \\
$\Delta$ Glycemia variation, pre and post 6MWT & -10.7 & \pm & 27.91 & 31.25 \\
& No & & $(\%)$ & $(33.3)$ \\
Lactate > (mmol/L) post 6MWT & 4 & & $(25.0)$ & \\
Test interrupted prematurely & 3 & &
\end{tabular}

Abbreviation: 6MWT, six-minute walk test; PE, perceived exertion scale; Max. Dist., maximum distance walked in meters; $\mathrm{HR}_{\max }$, maximum heart rate.

Parametric continuous data represented as mean \pm standard deviation; categorical data represented as NO, number of patients (\% of total).

cardiac condition this specific group has only $20 \%$ reduction in inspiratory muscle strengh and normal values in expiratory evaluation.

The analysis of the correlation of the variables obtained on the 6MWT only revealed an association of the distance walked and arrhythmia severity at the end of the 6MWT with EF (r=0.70 and -0.83 ) and $\mathrm{FC}(\mathrm{r}=0.73$ and 0.73$)(\mathrm{p}<0.05)$. Quality of life was moderately reduced $(31 \pm 3.33$ in a total of 105 points), but there was no correlation with the variables obtained on the 6MWT.

\section{Discussion}

The present study demonstrates that the 6MWT (without accompaniment) is a safe measure with regard to the hemodynamic and electrocardiographic response in patients with refractory heart failure. These findings furthermore reinforce the importance of a clinical evaluation during physical exertion in this patient population, as variables related to performance on the 6MWT appear to be associated to current clinical status and possibly with the cardiovascular prognosis. 


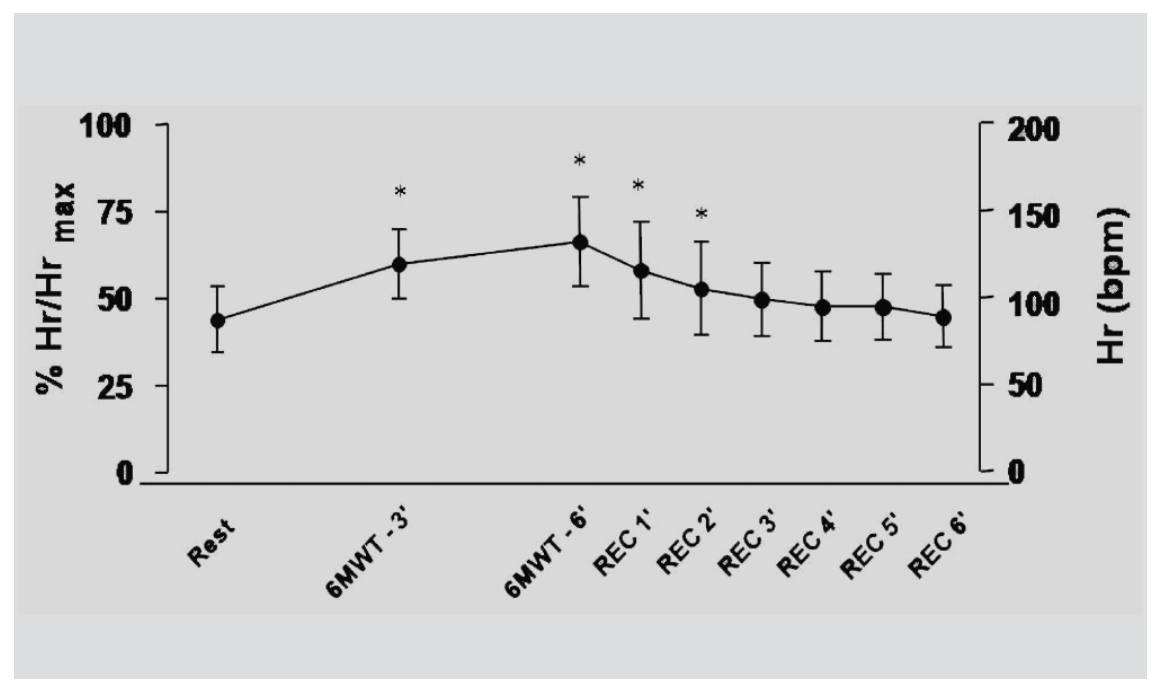

Figura 1. - Cardiovascular responses during 6MWT.

Abbreviations: 6MWT, six-minute walk test; REC, recovery period; HR, heart rate (bpm). Parametric continuous data represented as mean \pm standard deviation. Longitudinal analysis: ANOVA with DMS post hoc test. $(* \mathrm{p}<0.05)$.

The 6MWT is a simple, reproducible, low-cost exam with low operational difficulty and has important correlations with clinical variables such as exercise capacity, cardiopulmonary health, functional class, quality of life and prognosis [4-11, 17]. The present study was the first to use electrocardiogram telemetry monitoring during the unaccompanied 6MWT, thereby allowing the real-time determination of possible arrhythmia or signs suggestive of ischemia that may warrant test termination. A simplified analysis of peripheral lactate was also performed in order to gather information on alterations in the energy metabolism.

In the present study, quality of life was only moderately reduced and had no correlation with the variables measured during the 6MWT. This differs from the findings of preliminary studies demonstrating such a correlation. The discrepancy is likely due to the small number of patients and different functional classes in the present study.

The analysis of the occurrence of arrhythmia was carried out in three manners: The classification proposed by Low [18], which considered the severity of ventricular arrhythmia; the atrioventricular classification (AV), which divides arrhythmia into ventricular and supraventricular [11]; and the qualitative (descriptive) evaluation of the electrocardiogram findings [12]. The latter two report a greater incidence of arrhythmia in patients with a reduced oxygen supply in the myocardium. The analysis revealed a high probability of the occurrence of arrhythmia in this population. However, this condition was not exacerbated during the performance of the 6MWT, which suggests that safety of the method. Nonetheless, the need for clinical and preliminary dysfunction stratifying assessment must be emphasized. The routine use of an electrocardiogram system should be evaluated carefully, as the six-minute walk test is, in principle, a simplified test of easy reproducibility.

Heart rate was the variable that demonstrated the most expressive cardiovascular adjustment during the 6MWT. This response highlights its influence on the variation in the double heart product, which exhibits a loss in cardiovascular function in such patients, as evidenced by the reduced ventricular ejection fraction. Thus, as we could imagine, the chronothropic response was more important than the inotropic response in this population $[11,18]$. The $\mathrm{HRR}_{2}$ was apparently higher in the group with poor cardiac function. A number of studies have described a correlation between the behavior of heart rate recovery (HRR) and cardiovascular function, in which slower reductions are directly related to deterioration in cardiovascular function and mortality. This phenomenon is linked to an imbalance in the autonomic nervous system, generally produced by the over-stimulation of the sympathetic nervous system and a reduction in vagal activity, and measures changes in functional capacity following a conditioning program [8,9].

The simplified assessment of lactate allowed the estimation of additional information regarding the behavior of the energy metabolism during the walking test. Although the maximum heart rate $\left(\mathrm{HR}_{\max }\right)$ reached an average of just $66.3 \%$ of the predicted value, four of the patients surpassed $4 \mathrm{mmol} / \mathrm{L}$ in the analysis of lactate behavior, suggesting a greater use of the anaerobic metabolism [20]. Following the $6 \mathrm{MWT}$, the individuals reported an average perceived exertion of $14.3 \pm 1.55$ in a total of 20 points, which corresponds to exertion much closer to weariness and is compatible with the exertion reported in other studies. These findings allow us to infer a possible error in the estimation of maximum heart rate for these patients with an important functional limitation and most of whom $(83.3 \%)$ made use of $\beta$ blocker treatment.

In the initial clinical evaluation, body weight and muscle force production are important markers in this population. With the evolution of heart failure, these variables develop an increased metabolic energy demand associated to perceptible musculoskeletal degradation. This can generate a functional stage denominated cardiac cachexia, interfering negatively on cardiopulmonary conditioning and, consequently, on quality of life and cardiovascular prognosis $[2,3]$.

The analysis of the 6MWT correlation with clinical status primarily revealed that, despite pertaining to an apparently homogeneous group equally eligible for heart transplant surgery, the individuals exhibited diversified functional capacity, as estimated by the distance walked. This was especially true for the four individuals who walked distances of less than 300 meters. It is currently known that individuals with heart failure who travel less than 300 meters on the 6MWT have a much worse prognosis $[5,10]$. In the present study, the maximum distance walked 
on the 6MWT demonstrated an important correlation with classic clinical characteristics such as ejection fraction $(\mathrm{r}=0.70)$ and functional class $(\mathrm{r}=0.73)$. Although some studies question the use of the test as a measure of cardiovascular status, most randomized clinical trials are in favor of its diagnostic value in patients with heart failure [7-9].

The present study has limitations that should be addressed. We evaluated a relatively small sample of patients with an indication for elective heart transplant surgery. Although we have demonstrated relevant results in this study, we must stress the difficulty in access to this population. Thus, the population evaluated in the present study corresponded to $12 \%$ of the total number of patients registered in Sao Paulo state, which speaks to the importance of carrying out multi-center studies in the future.

In conclusion, the clinical and electrocardiogram behavior suggests that the six-minute walk test is safe, but may be considered of high intensity for some patients with severe heart failure. Variables related to performance on the 6MWT may be associated with clinical status.

Acknowledgements: Gerson Cipriano Jr ${ }^{1}$, Graziella França Bernardelli2, Ross Arena ${ }^{3}$, Luis Vicente Franco Oliveira $^{4}$, Enio Buffolo ${ }^{1}$, João Nelson Rodrigues Branco 2

${ }^{I}$ Physiotherapy Division, Faculty of Ceilândia, University of Brasília, UnB, Brasília, Brazil;

2 Cardiovascular Surgery Division, Department of Surgery, Federal University of Sao Paulo, Sao Paulo, Brazil;

3 Department of Physical Therapy, Box 980224 Virginia Commonwealth University/MCV Campus, Richmond, Virginia, USA;

${ }^{4}$ Rehabilitation Sciences Master's Program, Universidade Nove de Julho - UNINOVE, São Paulo, SP, Brazil.

Drs. Gerson; Graziella and Joao have contributed to (1) conception and design of the study; (2) analysis and interpretation of data; (3) writing the article or revising it critically for important intellectual content and (4) to prepare and approve the final version to be published. Dr. Isac de Castro, performed the (5) statistical analysis had full access to all of the data in the study, and takes responsibility for the integrity of the data and the accuracy of the data analysis. Dr. Enio, Ross and Luis have interpreted the (6) results and (7) help to prepare the first draft and the final version of the article.

\section{References}

1. Mady C. Current situation of the treatment of heart failure in Brazil. Arq Bras Cardiol 2007; 89: 84-6.
2. Anker SD, Coats AJ. Cardiac cachexia: a syndrome with impaired survival and immune and neuroendocrine activation. Chest 1999; 115: 836-47.

3. Juenger J, Schellberg D, Kraemer S, et al. Health related quality of life in patients with congestive heart failure: comparison with other chronic diseases and relation to functional variables. Heart 2002; 87: 235-41.

4. Myers J, Geiran O, Simonsen S, Ghuyoumi A, Gullestad L. Clinical and exercise test determinants of survival after cardiac transplantation. Chest 2003; 124: 2000-5.

5. Bettencourt P, Ferreira A, Dias P, et al. Predictors of prognosis in patients with stable mild to moderate heart failure. J Card Fail 2000; 6: 306-13.

6. Troosters T, Gosselink R, Decramer M. Six minute walking distance in healthy elderly subjects. Eur Respir $J$ 1999; 14: 270-4.

7. Delahaye N, Cohen-Solal A, Faraggi M, et al. Comparison of left ventricular responses to the six-minute walk test, stair climbing, and maximal upright bicycle exercise in patients with congestive heart failure due to idiopathic dilated cardiomyopathy. Am J Cardiol 1997; 80: 65-70.

8. Demers C, McKelvie RS, Negassa A, Yusuf S. Reliability, validity, and responsiveness of the six-minute walk test in patients with heart failure. Am Heart $J$ 2001; 142: 698-703.

9. Olsson LG, Swedberg K, Clark AL, Witte KK, Cleland JG. Six minute corridor walk test as an outcome measure for the assessment of treatment in randomized, blinded intervention trials of chronic heart failure: a systematic review. Eur Heart J 2005; 26: 778-93.

10. Bittner V. Determining prognosis in congestive heart failure: role of the 6-minute walk test. Am Heart J 1999; 138: 593-6.

11. Belardinelli R. Arrhythmias during acute and chronic exercise in chronic heart failure. Int J Cardiol 2003; 90: 213-8.

12. Galante A, Pietroiusti A, Cavazzini C, et al. Incidence and risk factors associated with cardiac arrhythmias during rehabilitation after coronary artery bypass surgery. Arch Phys Med Rehabil 2000; 81: 947-52.

13. Fisher JD. New York Heart Association Classification. Arch Intern Med 1972; 129: 836.

14. Rector TS, Cohn JN. Assessment of patient outcome with the Minnesota Living with Heart Failure questionnaire: reliability and validity during a randomized, doubleblind, placebo-controlled trial of pimobendan. Pimobendan Multicenter Research Group. Am Heart J 1992; 124: 1017-25.

15. AACVPR. Guidelines for Cardiac Rehabilitation and Secondary Prevention Programs. 3th ed. Champaing: $\mathrm{Hu}-$ man Kinetics; 2004. p. 288.

16. Borg GA. Psychophysical bases of perceived exertion. Med Sci Sports Exerc 1982; 14: 377-81.

17. Witham MD, McMurdo ME. Don't shoot the messenger: the 6-minute walk test is a useful outcome measure in exercise trials. Am Heart J 2003; 146: E7.

18. Lown B, Wolf M. Approaches to sudden death from coronary heart disease. Circulation 1971; 44: 130-42.

19. Wasserman K. The anaerobic threshold measurement in exercise testing. Clin Chest Med 1984; 5: 77-88. 